Journal of Mathematics and Informatics

Vol. 16, 2019, 23-39

ISSN: 2349-0632 (P), 2349-0640 (online)

Published 19 April 2019

www.researchmathsci.org

DOI: http://dx.doi.org/10.22457/jmi.138av16a3

Journal of

Mathematics and

Informatics

\title{
Human Iris Localization Combined with Ant Colony and Improved Hough Circle Detection
}

\author{
Jinhui Gong ${ }^{1}$, Guicang Zhang and Kai Wang \\ School of Mathematics and Statistics, Northwest Normal University \\ Lanzhou 730070, China \\ ${ }^{1}$ Corresponding author. Email: 321256487@qq.com
}

Received 5 March 2019; accepted 10 April 2019

\begin{abstract}
When the traditional Hough transform based on circle detection locates the human iris, it involves a three-dimensional parameter space, so there is a shortage of computational time and space overhead. Aiming at this problem, a Hough transform circle detection algorithm using gradient to reduce the spatial dimension of parameters is proposed. Firstly, the image is preprocessed by mathematical morphology to reduce noise and eyelash interference. Secondly, the ant colony optimization algorithm is used to preprocess the image. Edge extraction is performed to reduce the number of points participating in the Hough transform. Finally, the improved Hough transform is used to locate the iris. The high-quality and low-quality images are used to compare the traditional Hough transform method and the literature [13] method. The results show that the method not only improves the positioning speed, but also improves the positioning accuracy. Compared with other methods, the image quality is improved. The requirements are also significantly reduced.
\end{abstract}

Keywords: Iris localization; Hough transform; mathematical morphology; ant colony algorithm; edge detection

AMS Mathematics Subject Classification (2010): 62H35

\section{Introduction}

In the modern information network society, identity authentication is closely related to the daily life of individuals, such as aviation, finance, network, e-commerce, and national security. Because the traditional identity authentication method relies on external identity documents, user names, passwords and other factors, it is easy to be forgotten or easily 


\section{Jinhui Gong, Guicang Zhang and Kai Wang}

forged. Therefore, biometric recognition with the advantages of universality, uniqueness, stability, non-invasiveness, etc. [1] has become a trend of research and development, in which iris recognition has better security than fingerprint, palm print and face recognition[2]. Anti-deception and higher realism. Therefore, it is considered to be the most promising biometric method in academia and business [3-4].

The idea of using iris recognition was originally proposed by ophthalmologist Frank Birch in 1936. Daugman of the University of Cambridge invented the world's first human eye iris recognition machine, and in 1993 proposed the theoretical framework of iris recognition. The idea of using iris recognition was originally proposed by ophthalmologist Frank Birch in 1936. Daugman of the University of Cambridge invented the world's first human eye iris recognition machine, and in 1993 proposed the theoretical framework of iris recognition. There are mainly the circular calculus algorithm proposed by Daugman [5], the combination of edge detection and Hough transform proposed by Wildes [6]. In [7], an iris segmentation method based on image alignment is proposed. The corner angle detection method is used to detect the left corner of the eye. The batch alignment algorithm based on sparse low rank decomposition is used to process the image that has been classified. Compared with the iris positioning method, the method can effectively remove the noise formed by the occlusion, but the process is complicated and is not easy to implement. In 2007, Daugman [8-10] proposed that the active contour method is more suitable for describing the inner and outer boundaries of the iris than the fixed shape model, but the method is susceptible to interference from noise and occlusion, and converges to local minimum values, resulting in inaccurate positioning. Literature [11] proposed a Hough transform iris localization algorithm combined with morphology. In [12], the inner boundary of the iris is roughly positioned by the method of geometric gray projection. The improved Daugman operator is used to locate the outer boundary, and the inner and outer boundaries are fitted by the least squares fitting method. To split the iris. Zhu Lijun et al. proposed in the literature [13] to adopt the method of difference set and edge detection template to locate the inner and outer edges of non-ideal irises, but the algorithm does not take into account the interference of eyelashes and eye faces, and the algorithm is robust. Less sexual. In the literature [14], Liu Shuai et al. proposed an iris localization algorithm based on block search. First, the iris image is converted into a binary image by using the gray image of the iris image, and then coarsely positioned by the Hough transform circle detection method based on edge detection. The iris inner circle is out, but the Hough transform algorithm used is computationally intensive and difficult to implement. Xue Yadi, Wang Jinsong et al. in the literature [15] proposed the algorithm to approximate the radius compensation and center of the circle, and to locate the inner and outer edges of the iris. Ma Yide, Zhou Lijun proposed a vector field convolution VFC-based iris localization algorithm in the literature [16], which broke through the limitations of positioning the inner 
Human Iris Localization Combined with Ant Colony and Improved Hough Circle Detection

boundary of the iris on the basis of round fitting, and significantly improved the positioning accuracy. When the eyelashes and the eye face severely block the edge of the pupil and the spot is very close to the eye face, it may cause problems such as inaccurate positioning.

This paper mainly introduces an improved Hough transform circle detection iris location method of the traditional Hough transform based on circle detection to the human eye iris localization, due to the parameters involved in the space to achieve $3 \mathrm{~d}$, so there are large amount of calculation, the calculation is not convenient, slow speed is insufficient to solve these problems, such as a gradient is used to reduce parameter space dimension of the Hough transform circle detection algorithm, the original parameters of three-dimensional space to two-dimensional space, greatly reduce the amount of calculation, is conducive to improve the computing speed. First, this paper applies a mathematical morphology for iris image preprocessing, to reduce the noise and the interference of eyelash, secondly by using ant colony optimization algorithm [17] edge of iris image are extracted, and then to extract the image binarization, the use of iris's own characteristics, extract the pupil, estimate the radius of the iris, so as to estimate the approximate scope of the outside radius with the modified Hough transform circle detection algorithm for iris localization in the end Experimental results show that the human eye iris method adopts morphology and ant colony optimization algorithm to preprocess the human eye image, which greatly reduces the computation of subsequent Hough transform detection, and the threshold setting in the accumulation process increases the robustness of detection.

\section{Pretreatment}

The noise of the iris image of the human eye mainly comes from the eyelashes, and the eyelashes are scattered and non-directional fine-line objects. This characteristic is particularly suitable for the de-drying pretreatment of the original iris image using the morphological opening and closing operations.

In the morphology, the opening and closing operations are made up of corrosion and expansion. The closing operation is first expansion and then corrosion: the opening operation is first to etch and then expand. If you use a structural element $S$ pair to $A$ perform an open operation, Referred to as $A \circ S$, Can be expressed as

$$
A \circ S=(A \Theta S) \oplus S
$$

Therefore, using $S$ pair to $A$ do the opening operation is $S$ to $A$ perform the etching operation first, and then $S$ to expand the operation after the corrosion. In general, the open operation smoothes the outline of the image, breaking narrow connections and eliminating small burrs. The structural elements commonly used in the process are as follows: 
Jinhui Gong, Guicang Zhang and Kai Wang

$$
S=\left|\begin{array}{lll}
1 & 1 & 1 \\
1 & 1 & 1 \\
1 & 1 & 1
\end{array}\right|, S=\left|\begin{array}{lll}
1 & 0 & 0 \\
0 & 1 & 0 \\
0 & 0 & 1
\end{array}\right|, S=\left|\begin{array}{lll}
0 & 0 & 1 \\
0 & 1 & 0 \\
1 & 0 & 0
\end{array}\right|, S=\left|\begin{array}{lll}
0 & 1 & 0 \\
0 & 1 & 0 \\
0 & 1 & 0
\end{array}\right|, S=\left|\begin{array}{lll}
0 & 1 & 0 \\
1 & 0 & 1 \\
0 & 1 & 0
\end{array}\right|
$$

If the same applies $S$ to $A$ the structural element pair, Referred to as $A \circ S$, Can be expressed as

$$
A \bullet S=(A \oplus S) \Theta S
$$

Therefore, the closing operation of $S$ to $A$ is to use $S$ to perform the expansion operation on $A$ first, and then use $S$ to perform the corrosion operation on the expanded result. Since the eyelashes can be thought of as embedding some irregular gaps in the background of the image, the process of suppressing interference can be regarded as a process of "filling in" the gaps with patterns formed by structural elements. Obviously, the closed operation can also smooth the outline, but in contrast to the open operation, it can usually bridge narrow discontinuities and fill small holes.

Since the structural element is required to "fill in" the gap, the " 3 x 3 full "1" square matrix is selected as the structural element, and the morphologically preprocessed image is shown in FIG. The filtering results show that the noise in the image and the interference of the eyelashes are weakened to some extent.

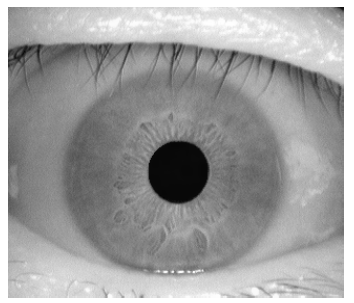

(a) original image

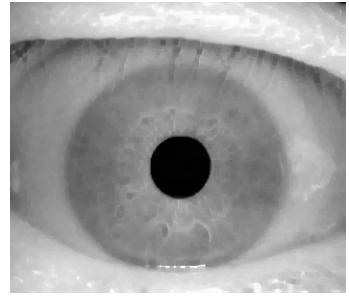

(b) morphologically filtered image

Figure 1: Morphological filtering preprocessing of the original image

In the comparison, we can't clearly see the difference between the processed images with the naked eye, but we can still see the changes at the edge of the image. In order to see the result of the processing, we compare the gray histograms before and after. as follows:

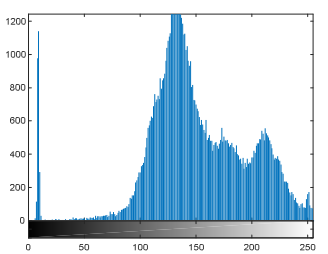

(a) Grayscale histogram of the original image

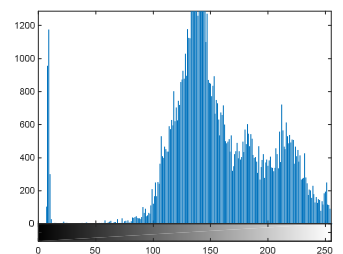

(b) Grayscale histogram after morphological processing

Figure 2: Comparison of grayscale histogram of image after original image and morphology processing. 
Human Iris Localization Combined with Ant Colony and Improved Hough Circle

Detection

\section{Using an improved ant colony optimization algorithm to detect iris edges}

The ant colony algorithm aims to find the optimal solution of the target problem by iterative iterative method. It is realized by searching the solution space by establishing pheromone information. We first abstract the iris image into an undirected graph, and then randomly place the ants in different positions in the graph. According to the search optimization process of the ant colony algorithm, the ant selects the iris edge point as the next moving target with a large probability. After many iterations of the loop, most of the ants are gathered on the edge of the iris to search for the edge information of the iris.

(1) Initialization process

Each of the pixels in the image is treated as a node by randomly placing $K$ only ants on the image of size $M \times N$. The initial value of each element of the pheromone matrix $\tau^{(0)}$ is set to $\tau_{\text {init }}$.

(2) Implementation process

In the first step of execution, one of the ants alone is randomly selected to allow the ant to move continuously on the image.

During the execution of the $n$ step, one of the ants from the top of $K$ the above is randomly selected to allow the ant to continuously move $L$ on the image. The ant moves from the node $(l, m)$ to the adjacent node $(i, j)$ according to a transformation probability defined by equation (3):

$$
p_{(l, m)(i, j)}^{(n)}=\frac{\left(\tau_{(l, m)(i, j)}^{n-1}\right)^{\alpha}\left(\eta_{(l, m)(i, j)}\right)^{\beta}}{\sum_{(i, j) \in \Omega_{(l, m)}}\left(\tau_{(l, m)(i, j)}^{n-1}\right)^{\alpha}\left(\eta_{(l, m)(i, j)}\right)^{\beta}}
$$

wherein $\tau_{(l, m)(i, j)}^{n-1}$ is the arc pheromone value of the linking node $(l, m)$ and the node $(i, j)$; The ant $a_{k}$ is located at the node $(l, m)$, and its $\Omega_{(l, m)}$ is its neighboring node; $\alpha, \beta$ indicating the importance of pheromone information and heuristic information; $\eta_{(l, m)(i, j)}$ indicates heuristic information from node $(l, m)$ to node $(i, j)$, and the value is fixed during the execution of each step.

(3) Update process

Using the classical chaotic system-Logistics map as a chaotic variable, iterate 


\section{Jinhui Gong, Guicang Zhang and Kai Wang}

according to equation (4).

$$
W_{i, j}^{(n+1)}=\mu W_{i, j}^{(n)}\left[1-W_{i, j}^{(n)}\right]
$$

In the formula, $n$ is the number of iterations, when $0 \leq W_{i, j}^{(n)} \leq 1, \mu=4$, the Logistics will be in a complete chaotic state. According to the permutation theory, each chaotic quantity is set to the pheromone value at one pixel, that is, the initial value of the pheromone matrix $\tau^{(0)}$ is based on the amount of chaos. When the pheromone matrix is updated, two update operations are performed.

The first pheromone update occurs after each ant has completed one step and the pheromone matrix is updated according to equation (5).

$$
\tau_{i, j}^{(n-1)}\left\{\begin{array}{c}
(1-\rho) \tau_{i, j}^{(n-1)}+\rho \cdot \Delta \tau_{i, j}^{(k)}+p W_{i, j}^{(n-1)}, \text { 若 }(i, j) \text { 被当前第k只蚂蚁访问时 } \\
\tau_{i, j}^{(n-1)}, \text { 其他 }
\end{array}\right.
$$

Where $\Delta \tau_{i, j}^{(k)}$ indicates the pheromone left by the $k$ ant only after passing through the pixel $(i, j)$, whose value is determined by the heuristic matrix $\eta_{i, j} ; W_{i, j}^{(n-1)}$ is a chaotic variable, (4) Iteratively obtained, $p$ is the coefficient; $\rho$ is the pheromone volatilization rate, which is improved by adaptive method:

$$
\rho^{(n)}=\left\{\begin{array}{c}
0.98 \cdot \rho^{(n-1)}, \rho \geq \rho_{\min } \\
\rho_{\min }, \rho<\rho_{\min }
\end{array}\right.
$$

First, establish a maximum pheromone volatilization rate $\rho_{\max }$ and minimum $\rho_{\min }$, and set $\rho_{\max }$ as the initial value of $\rho$. After each iteration, the pheromone volatilization rate will be according to the formula (6) Update.

The second pheromone update occurs after all the ants have completed the iteration in each step and is updated according to (7).

$$
\tau^{(n)}=(1-\varphi) \cdot \tau^{(n-1)}+\varphi \cdot \tau^{(0)}
$$

As the number of iterations increases, the pheromone will gradually evaporate, causing a large number of ants to stagnate, which directly affects the efficiency of the ant colony algorithm. In order to prevent a large number of ants from stagnation due to the volatilization of pheromone, the pheromone value of each point is controlled to a certain range on each path by manual intervention when the pheromone is updated for the second 
Human Iris Localization Combined with Ant Colony and Improved Hough Circle Detection

time. Inside, ie $\tau_{i, j} \in\left[\tau_{\min }, \tau_{\max }\right]$, the specific practices are as follows:

$$
\tau_{i, j}^{(n)}=\left\{\begin{array}{c}
\tau_{\min }, \tau_{i, j}^{(n)} \leq \tau_{\min } \\
\tau_{i, j}^{(n)}, \quad \tau_{\min }<\tau_{i, j}^{(n)}<\tau_{\max } \\
\tau_{\max }, \tau_{i, j}^{(n)} \geq \tau_{\max }
\end{array}\right.
$$

Separate the pupil and estimate the inner radius of the iris

Careful observation of the eye image (see Figure 1(a)) reveals that the pupil is much darker than the rest of the eye, so we can use the binarization method to separate the pupil and estimate the inner radius of the iris based on the area of the pupil image. The most critical point of the binarization method is how to choose the threshold [18].The specific process is: first calculate the gray histogram of the entire image. It has two main peaks. The first peak corresponds to the gray-concentrated area of the pupil, and the second peak corresponds to the gray-concentrated area of the iris. Obviously, the selection of the binarization threshold for extracting the pupil should be on the right side of the first peak (see Figure 2). Figure 3 shows the results of binarization separation under different thresholds. In Figure (a), the threshold value is 150 . It can be seen that there is too much iris information in the separation result. The iris and the pupil are mixed together, and the effect is not ideal. Take "threshold value $=120$ ", compared with the map (a) iris information interference is much reduced, can clearly identify the pupil, but there are still interference between the eyelashes and the eye curtain in the separation result; Figure (c) takes "threshold value $=60$ ", It just fits to the right of the first peak of the histogram. It can be seen that the separation result is ideal when "Threshold $=60$ " is selected.

Let the function of the extracted pupil image be $p(i, j)$, select the appropriate threshold $T$, and let:

$$
f(i, j)=\left\{\begin{array}{cc}
0 & 0<p(i, j)<T \\
1 & p(i, j)>T
\end{array}\right.
$$

Thus, the area of the pupil is determined as:

$$
S=\sum_{(i, j) \in I} f(i, j)
$$

Estimate the radius of the pupil: 
Jinhui Gong, Guicang Zhang and Kai Wang

$$
r=\sqrt{S / \pi}
$$

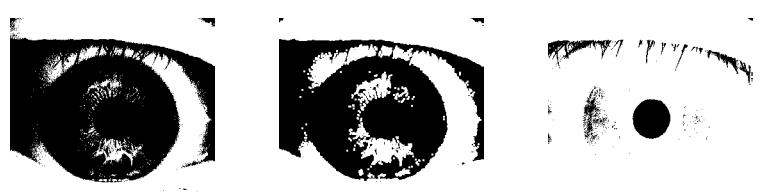

(a) Threshold $=150$

Binarization Binary image morphology Separate pupil drying treatment

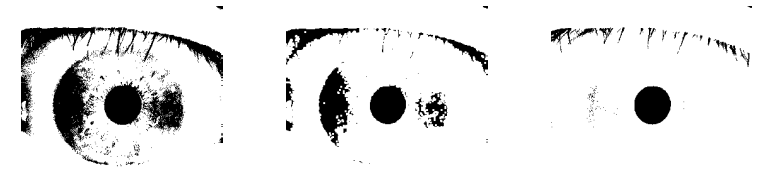

(b) Threshold $=120$

Binarization Binary image morphology Separate pupil drying treatment

(c) Threshold $=60$

Binarization Binary image morphology Separate pupil drying treatment

Figure 3: Binary separation results at different thresholds

\section{Improved Hough transform algorithm to locate the inner and outer edges of the} iris

\subsection{Traditional Hough transform principle based on circle detection}

We want to detect and determine the circle on the XY plane, generally let $\left\{\left(x_{i}, y_{i}\right): i=\right.$ $1,2, \ldots, n\}$ be the set of points on the circle given in the image, $(x, y)$ is any point in the set, in the parameter system $(a, b, r)$, this point The equation is:

$$
(a-x)^{2}+(b-y)^{2}=r^{2}
$$

If the unknowns in equation (12) are converted to parameters, $x-y$ is replaced by a 
Human Iris Localization Combined with Ant Colony and Improved Hough Circle Detection

constant, and $a-b-r$ is replaced by an unknown quantity, then equation (12) is a cone equation. In other words, the fixed point on a certain circle in the $\quad x-y$ plane is mapped to the three-dimensional cone in the parameter space. In this way, the detection problem of the circle on the $x-y$ plane is transformed into the intersection of the three-dimensional cone on the detection $a-b-r$ parameter space. The equation of the image plane is transformed into the parameter space as shown in Figure 4 below.

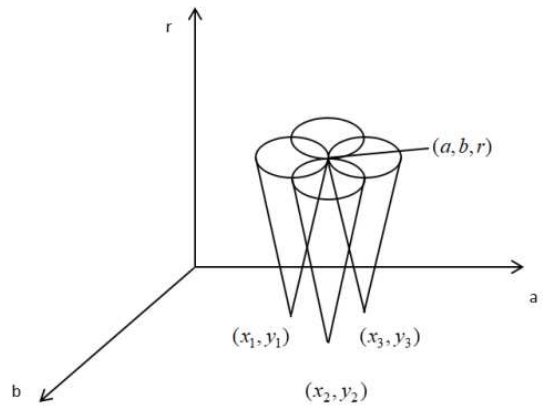

Figure 4: Parameter space representation of the circle

The parameter space can be considered discrete when it is calculated. Design a three-dimensional accumulation array $A(a, b, r)$, the calculation process is to let $a, b$ increase in the range of values, solve the $r$ value that satisfies the formula (6), each time a $(a, b, r)$ value is calculated, the array element is $A(a, b, r)$ Add 1, i.e. $A(a, b, r)=$ $A(a, b, r+1)$. After the calculation is completed, the largest $A(a, b, r)$ corresponding to the $a, b, r$ is the parameter of the circle sought.

It can be seen that the algorithm complexity of the above method is too high. If we want to detect small images of $100 \times 100$ resolution, we need at least the parameter space of $100 \times 100 \times 100$ size. The method of directly using the parameter space voting is complicated, the resource demand is large, and the processing time is long. In the case of large noise and complex image backgrounds, a large number of useless points will also participate in voting, which greatly reduces the performance of the algorithm and even affects the detection results.

\subsection{Improved Hough transform algorithm for detection of inner and outer circles of} iris

It can be seen from the above analysis that the key to improving the efficiency of Hough transform is to reduce the number of points participating in the Hough transform and reduce the dimension of the cumulative array as much as possible. Here we use the gradient method to reduce the dimension of the cumulative matrix, the specific process is 
as follows:

If the given point in the original image space is determined by the gradient, the gradient direction information obtained in the gradient calculation can be utilized to reduce the amount of computation required to obtain the Hough transform. Let the radius $r$ of the circle be known to convert the problem into a two-dimensional parameter space, as shown in Figure 5. The original parametric trajectory is on the entire conical surface. If $r$ is known, the trajectory parameter is the perimeter of a circle with a radius of $r$. Here, the boundary in the image space and the trajectory in the parameter space are both circles, so here is the circle-circumference pair.
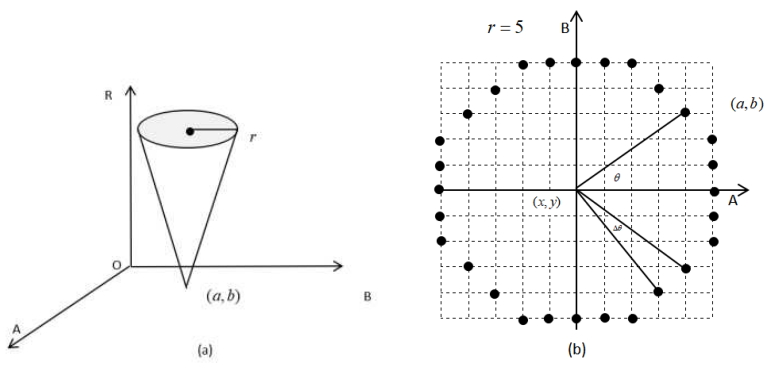

Figure 5: Utilization of gradient information in circle detection

For a circular point $(x, y)$ of the origin of the coordinate system in Figure (b), the trajectory of its center is shown by the small black dot (here $r=5$ ). If we first divide the formula (12) into $x$ and substituting it into $\frac{d y}{d x}=\tan \left(\theta-\frac{\pi}{2}\right)$ (where $\theta$ is the angle between the gradient direction and the $A$ axis positive direction), then the differential result is combined with the formula (12) and Solve for $a, b$ (this result can also be obtained from Figure (b)):

$$
\begin{aligned}
& a=x-r \sin \theta \\
& b=y+r \cos \theta
\end{aligned}
$$

It can be seen from the above formula that if the gradient direction is known, then $a$ and $b$ can be directly obtained for each circumferential point, instead of having $a$ take all possible values on the $A$ axis to calculate. This can be further explained with the aid of Figure 6. Figure (a) shows the case where the gradient direction is not considered. The small dots in the figure correspond to the circumferential points in the image space, and the thin line circles are all possible center positions. At this point, since it is not known where the center of the circle should be, it is necessary to check the circumference of the entire thin line to determine the center point in the image space. Figure (b) shows 


\section{Human Iris Localization Combined with Ant Colony and Improved Hough Circle}

Detection

the case where the gradient direction is considered, and the arrow in the small circle corresponding to the circumferential point in the image space in the figure represents the gradient direction. After considering the gradient pointing, the possible center position is greatly reduced. In other words, it is only necessary to consider a part of the thin line arc in the direction indicated by the gradient (because it is a digital image, the arc has a certain length). The length of this arc is only the $\frac{\Delta \theta}{2 \pi}$ of the entire circumference (as shown in Figure 6(b)).

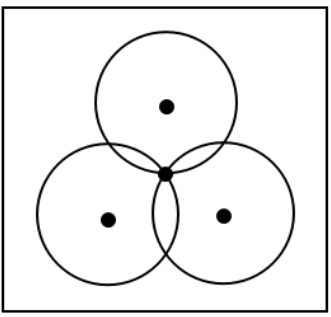

(a)

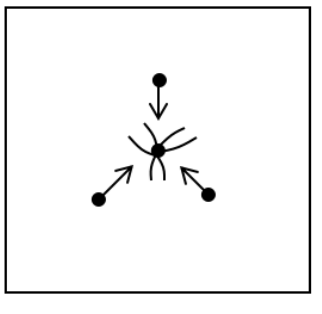

(b)

Figure 6: Shows the cumulative array in the case of gradient or not

\subsection{Algorithm steps}

Step 1: preprocessing: Here we use the morphological closed operation to process the original iris image, specifically: select the " $3 \times 3$ "full' 1 'square matrix as the structural element, and then call the imdilate and ierode functions to realize the closed operation.

Step 2: edge detection: Since the Hough transform can only process binary images, it is generally necessary to perform edge detection on the image before performing the transformation. Here we use the improved ant colony optimization algorithm for edge detection. The specific detection steps are as follows:

(1) Initialization process

Firstly, the pre-processed image size is divided into parts, and then the gradient value of each pixel point and the regional gray mean value difference are respectively calculated to obtain a combined gradient value; the ants are allocated according to the size of each small area combined with the gradient sum; Then, the ants assigned to each small area are randomly sent to the small area; then the heuristic matrix is established according to the Logistics chaotic system; finally all parameters are initialized.

(2) Calculate the probability transfer function

The ant's next search position is determined by calculating the probability in the probability transfer function. 
(3) pheromone update

Whenever an ant completes an iteration, the pheromone is updated according to equation (5) and the iteration of the parameter $\rho$ and the chaotic variable $W$ is completed. After the first update is completed, the second update of the pheromone is performed according to equations (7) and (8).

(4) Testing

After all the ants have completed the iteration, the final pheromone matrix $\tau^{[N]}$ is used to determine whether each pixel is an edge. Here, the adaptive Otsu threshold method [21] is used to adaptively select a reasonable threshold $T_{y u}$ from the pheromone matrix and compare it with each element in the pheromone matrix. If the pixel in the pheromone matrix is $(i, j)$. The pheromone value is greater than the threshold $T_{y u}$ then $(i, j)$ is the edge point, and vice versa, the resulting iris edge image is shown in Figure 7.

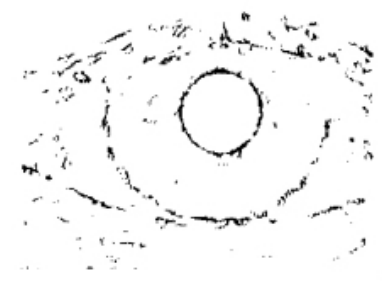

Figure 7: Iris edge detected by ant colony algorithm

Step 3: binarization and extraction of pupils

Step 4: Hough transform detection.

The extracted iris edges are compiled into a reference table, and the gradient angle $\phi_{i} \phi_{i}$ is calculated for each edge point, and for each gradient angle $\phi_{i}$, the distance $r_{i}$ and angle $\alpha_{i}$ corresponding to the reference point are calculated.

Create a two-dimensional cumulative array $A(a, b)$ in the parameter space, the initial value is 0 . For each point on the edge, calculate the gradient angle at that point. Then, calculate the position value of each possible reference point by equation (13) and add 1 to the corresponding array element $A(a, b)$.

After the calculation is completed, the $A(a, b)$ value corresponding to the array element having the largest value $a, b$ is the reference point sought in the image coordinate space, and the detection ends.

Step 5: In order to make the detection result have certain robustness when the iris image is deformed, and get the correct detection result, it is necessary to compare the number of edge points on the circle with a given threshold $T$, If the number of edge points on the circle is greater than the given threshold $T$, the solution will be completed; 
Human Iris Localization Combined with Ant Colony and Improved Hough Circle Detection

otherwise, let $r=r-1$, then return to step four calculations.

\section{Experimental results and analysis}

The computer model used in the simulation experiment was Lenovo G40, which was configured with CPU $2.10 \mathrm{GHz}$ and $8 \mathrm{~GB}$ of memory. The experimental environment was Matlab R2016a and Windows 10. In this paper, the iris images of the iris database CASIA-IrisV1.0[20], CASIA-Iris-Lamp[21] and Device2 provided by the Institute of Automation of Chinese Academy of Sciences were tested. The CASIA-IrisV1.0 library includes 756 eye images of 108 subjects, each with 7 8-bit grayscale images, and the resolution is, the library is a high quality image library. The image in the CASIA-Iris-Lamp library incorporates illumination interference, and the image pixel size is . The library is a poor quality image library. Device2 is a low quality image library. Figure 8 is a diagram showing the results of iris positioning in images of three different libraries respectively extracted,After many trials, the final selection parameters [24]are $\alpha=1, \beta=0.1, \tau_{\min }=0.01, \tau_{\max }=10, \rho_{\max }=0.95, \rho_{\min }=0.01, p=0.002$,step size $L=400, m=300$. It can be seen from the figure that the iris is successfully located using the algorithm of this paper, whether it is a high-quality map or an interference graph, which indicates the breadth of the algorithm.

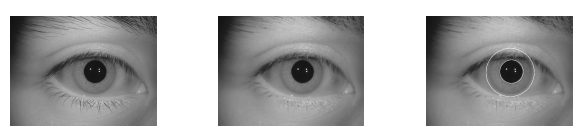

Image in CASIA Preprocessed Test results Iris positioning results -Iris-Lamp library image
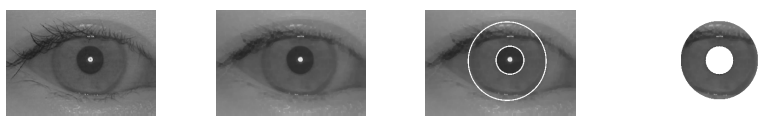

Image in the Preprocessed Test results Iris positioning results

Device2 library image

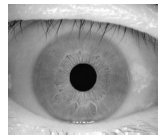

Image in CASIA Preprocessed

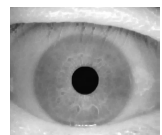

-Iris V1.0 library

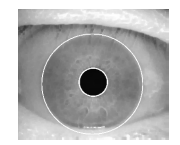

Test results

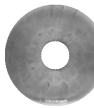

Iris positioning results

Figure 8: Image detection results in different libraries

Experiments were carried out on the traditional Hough transform method, the method in [13] and the method in this paper. The results are compared as shown in Fig. 9. 
Jinhui Gong, Guicang Zhang and Kai Wang

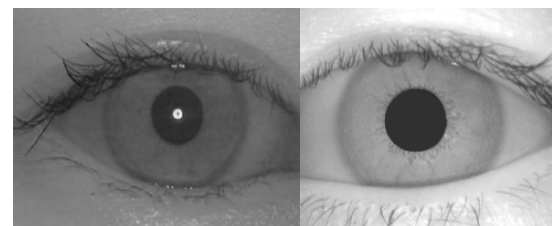

(a) Image in Device2 and CASIA-IrisV1.0 library

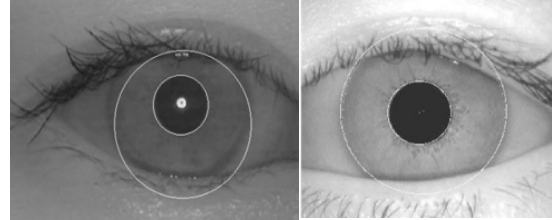

(b) Traditional Hough transform test results

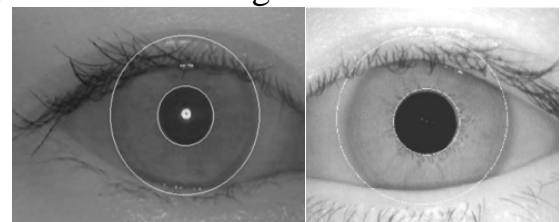

(c) Document [13] algorithm test results

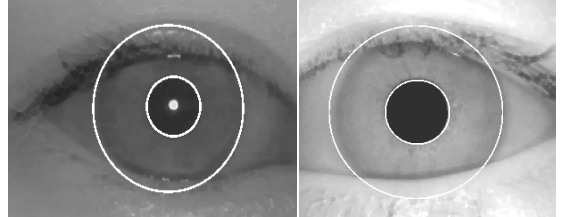

(d) Algorithm detection results in this paper

Figure 9: Comparison of iris positioning results

It can be seen that the traditional Hough transform has higher image quality requirements and is susceptible to interference from eyelashes and noise. When the picture quality is poor, the accuracy of positioning is significantly reduced. In the literature [13], the morphological method is combined for iris positioning. However, the accuracy of the positioning of the outer circle of the iris is still lacking. In contrast, the algorithm not only reduces the interference of eyelashes, noise, etc. in the preprocessing stage, but also the image quality requirements are significantly reduced, regardless of the high quality. Or low-quality images can be well positioned, and the positioning of the inner and outer circles of the iris is more accurate and clear.

In order to further verify the effectiveness of the iris localization algorithm, the running time of different algorithms is given, as shown in Table 1; and the accuracy bias [19] (Accuracy error, AE) is introduced to measure the performance of the algorithm. As shown in Table 2. defined as:

$$
A E=\frac{\left|N_{a c t}-N_{\text {det }}\right|}{N_{\text {act }}} \times 100
$$


Human Iris Localization Combined with Ant Colony and Improved Hough Circle Detection

Here $N_{a c t}$ and $N_{\text {det }}$ are the actual iris area and the number of pixels contained in the detected iris area, respectively. We manually calculated the actual iris pixels, and the number of pixels in the detected iris area can be obtained by a simple line main scanning method. If $A E$ is less than $10 \%$, then the detected iris is considered a true iris. Accuracy Rate (AR)[23] is defined as:

$$
A R=\frac{N_{\text {success }}}{N_{\text {total }}} \times 100
$$

where $N_{\text {success }}$ is the total number of eyes that the iris has successfully positioned, $N_{\text {total }}$ is the total number of images in the database.

Table 1: Comparison of running time of each algorithm (S)

\begin{tabular}{cccc}
\hline Image & $\begin{array}{c}\text { Traditional Hough } \\
\text { transform }\end{array}$ & $\begin{array}{c}\text { Literature } \\
{[13]}\end{array}$ & $\begin{array}{c}\text { This } \\
\text { article }\end{array}$ \\
\hline Device2 library image & 4.016 & 3.672 & 1.297 \\
$\begin{array}{c}\text { CASIA-IrisV 1.0 library } \\
\text { image }\end{array}$ & 3.516 & 2.413 & 1.012 \\
\hline
\end{tabular}

Table 2: Comparison of positioning accuracy of each algorithm (\%)

\begin{tabular}{cccc}
\hline image & $\begin{array}{c}\text { Traditional Hough } \\
\text { transform }\end{array}$ & $\begin{array}{c}\text { Literature } \\
{[13]}\end{array}$ & $\begin{array}{c}\text { This } \\
\text { article }\end{array}$ \\
\hline $\begin{array}{c}\text { Device2 library image } \\
\text { CASIA-IrisV 1.0 library } \\
\text { image }\end{array}$ & 79.0 & 86.8 & 92.8 \\
\hline
\end{tabular}

In summary, the proposed algorithm has significantly improved runtime and positioning accuracy compared to the traditional Hough transform algorithm and the algorithm given in [13]. Therefore, the algorithm is an effective iris localization algorithm.

\section{Conclusion}

In this paper, the traditional iris localization algorithm based on Hough transform circle detection is improved. Firstly, the improved ant colony optimization algorithm is used to extract the edge of the iris image, which reduces the number of points participating in the 
Jinhui Gong, Guicang Zhang and Kai Wang

Hough transform. Secondly, the circle in the $x-y$ space is transformed into the parameter space, and the dimension information is used in the parameter space to reduce the dimension of the cumulative matrix, thereby reducing the computational complexity of the Hough transform. Finally, the improved Hough transform is used to detect the inner and outer boundaries of the iris and accurately locate the iris. The article also used the iris images of CASIA-IrisV1.0, CASIA-Iris-Lamp and Device2 provided by the Institute of Automation of Chinese Academy of Sciences to carry out a large number of experiments, which proved the feasibility of the improved algorithm and achieved good results. Due to factors such as equipment, the human eye image is subject to interference from light, noise, eyelashes, and eye faces during the acquisition process. Therefore, the quality of the captured image is sometimes poor, and the precise positioning of the image that is seriously disturbed is the next. Further research.

Acknowledgment. The authors acknowledge the following organization for supporting their research.

a) National natural science foundation of China (project number: 61861040);

b) Science and technology project funding of gansu province (projectNO.17YF1FA119);

c) Lanzhou science and technology planning project (project number: 2018-4-35).

Also, the authors are grateful to the reviewers for their suggestions for improvement of the paper.

\section{REFERENCES}

1. Wang Yonghong, Zhu Yu and Tan Tiebei, Biometric recognition based on iris pattern, Acta Automatica Sinica, 28(1) 92002) 1-10.

2. Yang Yang, Cong Yu, Hang xiao and Nan-xiang Yu, A study of recognition about human face, Journal of Mathematics and Informatics, (2017) 25-35.

3. Xu Lin, Research on several key issues of iris recognition, Shenyang: Shenyang University of Technology, 2008.

4. L.L.Pan, Research on Iris recognition theory, Hefei: University of Electronic Science and Technology of China, 2012(Ch).

5. Daugman I, How iris recognition works, IEEE, 14(1) (2004) 21-30.

6. R.Wildes, Iris recognition: An emerging, biometric techno logy, Proceeding of IEEE, 85(9) (1997) 1348-1363.

7. Zeng Ye, Cai Biye, Song Yun, et al., An iris segmentation method based on image alignment, Computer Engineering, 7 (2015) 203-213.

8. J.Daugman, How iris recognition works, IEEE Trans Circuits and Syst for Video Tech, 14(1) (2004) 21-30 
Human Iris Localization Combined with Ant Colony and Improved Hough Circle Detection

9. J.Daugman, Demodulation by complex-valued wavelets for stochastic pattern recognition, Int J Wavelets, Multiresolution Inf Processing, Singapore: World Scientific Publishing Co, 2003: 1-17.

10. J.Daugman, New methods in iris recognition, IEEE Transactions on Systems, Man, and Cybernetics, Part B: Cybernetics, 37(5) (2007) 1167-1175.

11. Tian Xu-zi, Zhang Yuan and Huang Li-yu, Improved Hough transform iris location algorithm based on morphology, Computer Engineering and Applications, 48(12): (2012) 179-182.

12. Xu Wei, Quan Fangfang, Jiang Yuanyuan, et al, Application of improved algorithm in iris recognition, Computer Technology and Development, 23(3) (2013) 34-37.

13. Zhu Lijun and Yuan Yuqi, Non-ideal iris localization based on sum difference and edge detection template, Journal of Computer Applications, 6 (2018) 38-56.

14. Liu Shuai, Liu Yuanning, Zhu Xiaodong, et al, Iris localization algorithm based on block search, Computer Engineering and Applications, 54(18) (2018).

15. Xue Yadi, Wang Jinsong, Fan Chunyi, Iris boundary location method based on quadratic circumferential compensation, Advances in Laser and Optoelectronics, 5 (2018) 25.

16. Ma Yide and Zhou Lijun, Iris localization based on vector field convolution, Infrared and Laser Engineering, 10 (2014) 43.

17. Wang Kai and Zhang Guicang, Image edge detection based on improved ant colony algorithm, Computer Engineering and Applications, 53 (2017 ) 23.

18. Zhan Yan, Wei Changfa, Liu Qingping, et al, Research on iris image preprocessing based on Hough transform, Software Guide, 10 (2016) 15.

19. M.T.lbrahim, T.M.Knan, S.A.Knan, et al, lris localization using local histogram and other image statistics, Optics and Lasers in Engineering, 50 (5) (2012) 645-654.

20. Institute of Autom ation, Chinese Academy of Sciences, ASIA iris image database (ver 1.0) [EB/OL], [2010-10-12], www.sinobiom etrics.com.

21. CASIA,CASIA-Iris-Image-Database-Version4.0[EB/OL],(2013-05-13),http://biometr ics,idealtest.org.

22. Y.J.Zhang. A survey on evaluation methods for image segmentation, Pattern Recognition, 29(8) (1996) 1335-1346.

23. Hong-jie Li, Gui-cang Zhang and Zong-yuan Zhu, Color image segmentation based on PCNN, Journal of Mathematics and Informatics, (2018) 41-53.

24. Jin-Hua Chen, Xiang-Lian Yu, Xiao-Guang Lv, Shun-Xu Wang and Le Jiang, Augmented Lagrangian method for image restoration with spatially adapted parameter selection, Journal of Mathematics and Informatics, 14 (2013) 34-42. 